\title{
Development of 3D Measuring Techniques for the Analysis of Facial Soft Tissue Change
}

\author{
Z. $\mathrm{Mao}^{1}$, P. Sebert ${ }^{1}$, and A.F. Ayoub ${ }^{2}$ \\ 1 The University of Glasgow, Glasgow, Scotland, UK \\ 2 Glasgow Dental Hospital and School, Glasgow, Scotland, UK
}

\begin{abstract}
Three-dimensional facial information is very important for assessing the influence of clef lip repair and measuring the facial growth between cleft and non-cleft children. In this paper, 3D techniques for measuring facial soft tissue change and extracting useful 3D shape information are presented. Firstly, a robust 3D registration algorithm which combines landmark-based and surface-based registration techniques is described. It uses a new surface-based registration algorithm - HICP algorithm to refine landmark-based alignment. We then describe a graphical user interface for manually extracting 3D facial landmarks. Experimental tests on both simulated surface data and real facial scans have been carried out to validate the HICP algorithm.
\end{abstract}

\section{Introduction}

Ora-facial clefting is the most common birth defect in the cranio-facial region. The main challenge posed by cleft lip and palate is the achievement of an early morphological and functional repair of the affected structures and to maintain the normal development of affected children. Two research projects, funded by the chief Scientists Office of the Scottish Executive and the National Lottery Board through the Cleft Lip and Palate Association, are currently being run by the Departments of Oral Surgery, Computing Science and Statistics at the University of Glasgow. The studies involve capturing multiple 3D images of both cleft and normal children at different stages throughout the first five years of life. The overall aim of the research is to undertake advanced morphometric assessment of non-cleft, cleft, and surgically-managed cleft patients to assess the influence of surgical lip repair on facial morphology. This will be achieved through the development of 3D-based surface imaging, anatomy and analysis techniques.

This paper presents a robust 3D registration technique after a brief description of the 3D imaging system for capturing facial models. The registration algorithm combines landmark-based and surface-based registration techniques and uses a modified Iterative Closest Point (ICP) [1] algorithm to refine landmarkbased alignment. Facial soft tissue change in terms of surface area and soft tissue volume, before and after surgical treatment can then be measured based on the aligned models. A user-friendly graphical interface is developed for manually extracting 3D facial landmarks for statistical shape analysis [2]. The registration 
algorithm is validated by experimental tests on both simulated surface data and real facial scans.

\section{3D Facial Data Acquisition System}

The human face is a three-dimensional object, therefore accurate 3D information of facial morpholgy is very important for auditing surgical outcome of cleft lip repair and measuring facial growth. The 3D image acquisition system employed in the research projects is the C3D [3] system developed by the collaboration between The Turing Institute and Glasgow University.

In this system, a pair of video cameras is placed at each side of the patient's face, which then takes a stereo picture. The cameras and the light source are angled at 60 degrees at a distance of 1.75 meters from the patient. A computercontrolled texture flash projector illuminates the subject with random texture pattern to facilitate stereo matching. The image capture time is about 30 millisecond and a personal computer produces the 3D facial model. Detailed system description can be found in [3]. C3D system has the following characteristics which suit this application.

- Fast facial surface capture, approximately 30 ms, especially suitable for capturing young children's faces.

- High resolution cameras: 1000 x 800 pixels.

- Accuracy of localising facial landmarks to $0.5 \mathrm{~mm}$.

- No exposure to harmful radiation and therefore suitable for routine use.

- Simple input operation and quick 3D display.

Fig 1 shows a cleft child's face captured by the C3D system. The 3D facial model is represented by triangle meshes or $3 \mathrm{D}$ surface points which can be directly used for measurement and model registration. This system has already been installed in Glasgow Dental Hospital and Yorkhill Hospital for Sick Children and the data collection is in progress.

\section{Method}

Techniques in image processing, computer vision and statistical shape analysis are being developed to provide the facility for measuring facial configurations in three dimensions. These techniques include registering facial models, measuring surface area and volume difference, extracting facial landmarks, performing statistical-based shape analysis and so on.

\subsection{Measure Facial Soft Tissue Changes}

To conduct the assessment of facial soft tissue changes, the change of surface area and soft tissue volume, before and after surgical treatment, a robust 3D registration technique has been developed to align two facial models captured at 


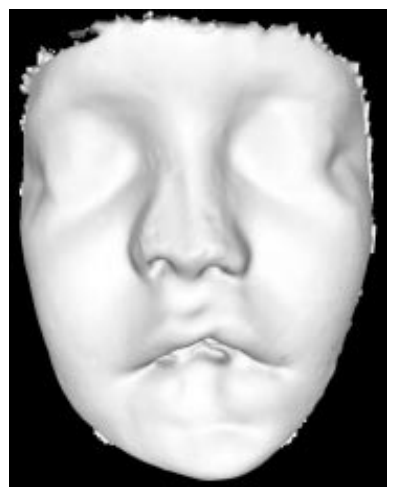

(a) Shaded facial surface.

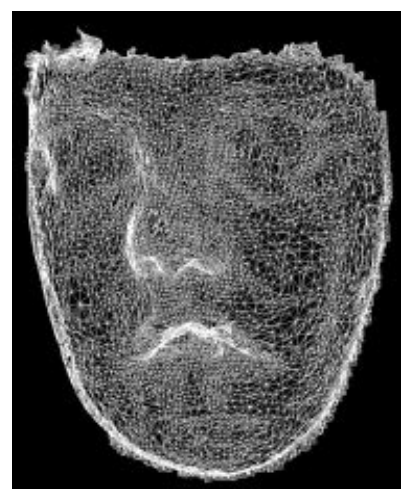

(b) Facial surface displayed by triangle mesh.

Fig. 1. 3D cleft facial model captured by C3D system.

different times. This algorithm is a combination of landmark-based and surfacebased registration techniques.

Firstly, corresponding 3D facial landmarks are manually extracted from two models using a purpose-built graphical interface (see 3.2). Landmark-based registration is then carried out to compute the relative rotation and translation between two models using a Singular Value Decomposition (SVD) approach 44. The computed transformation is used as the initial guess in the following surfacebased registration.

The surface-based registration algorithm we developed is a modified version of ICP 1 algorithm and directly uses the 3D surface points captured from the C3D system to calculate the pose parameters. It shares the similar idea to ICP algorithm which is to register a $3 \mathrm{D}$ point set $P$ having $N_{p}$ points $\left\{p_{i}\right\}, i=$ $1, \ldots, N_{p}$ with a second $3 \mathrm{D}$ point set $M=\left\{m_{i}\right\}, i=1, \ldots, N_{m}$ by calculating the closest point between an individual point $p_{i}$ and $M$ based on the Euclidean distance metric. After the set of closest points has been computed, a rigid body transformation is determined which minimises the equation

$$
\sum^{2}=\sum_{i=1}^{N}\left\|m_{i}-\left(R p_{i}+T\right)^{2}\right\|
$$

where $p_{i}$ is a $3 \mathrm{D}$ point with $x-, y-$ and $z$-coordinate in the first point set and $m_{i}$ is its closest point in the second point set; $R$ represents rotation matrix and $T$ is the translation vector. The registration $F(R, T)$ is then applied to $\left\{p_{i}\right\}$ and the process repeated until the change in mean square error falls below a pre-set tolerance. 
The difference between our method and the original ICP algorithm is that our algorithm considers outlier problem and instead of using all the closest point pairs obtained in each iteration for pose $(R, T)$ estimation, a weight $w_{i}$ is computed for every closest point pair. Pose estimation is then computed by minimising

$$
\sum^{2}=\sum_{i=1}^{N} w_{i}\left\|m_{i}-\left(R p_{i}+T\right)\right\|^{2}
$$

The method for calculating weight $w_{i}$ can be expressed as

$$
w_{i}=\left\{\begin{array}{l}
{\left[1-\frac{\left\|\varepsilon_{i}\right\|^{2}}{(c S)^{2}}\right]^{2} \quad \text { if }\left\|\varepsilon_{i}\right\| \leq c S ;} \\
\text { otherwise }
\end{array}\right.
$$

where $\varepsilon_{i}=m_{i}-\left(R p_{i}+T\right)$, the residual error (distance between closest point pair); $S$ is a scale estimator that is set to median of absolute distance and $c$ is a tuning constant which was set to 6 as suggested in [5].

Equation (3) was firstly presented by Haralick et al [5] for estimating pose parameters when the point correspondences between two data sets are known and hence we name our surface registration algorithm HICP. Our contribution in developing HICP algorithm is that we generalized the weighted least-squares techniques to iteratively locating the point correspondences and then calculating the transformation parameters and updating the data. The original design of the weighted least-squares algorithm [5] assumes that the point correspondence is known while in our algorithm a priori point correspondence is not required. Another difference from the original ICP algorithm is that a SVD algorithm [4] was used to calculate the transformation rather than using quaternions and accordingly reduces the complexity of the algorithm.

After models are properly aligned using the proposed method, soft tissue changes in surface area and volume can be measured. This registration technique can be further applied to determine facial symmetry plane [6].

\subsection{Facial Landmark Extraction}

A user-friendly software package has been developed to manually extract homologous facial landmarks that are required for both statistical shape analysis and approximate registration prior to applying the HICP algorithm to achieve close registration. This software is capable of displaying multiple facial models from different viewpoints at the same time. Users can easily move, zoom or rotate the facial models to a convenient position when placing landmarks. Fig 2 shows an example of the software interface. Circles indicate the positions of landmarks and vectors give the direction of surface normals at this landmark. The procedure of landmark detection can be further automated by using Active Shape Model (ASM), snakes or curvature-based algorithm. 


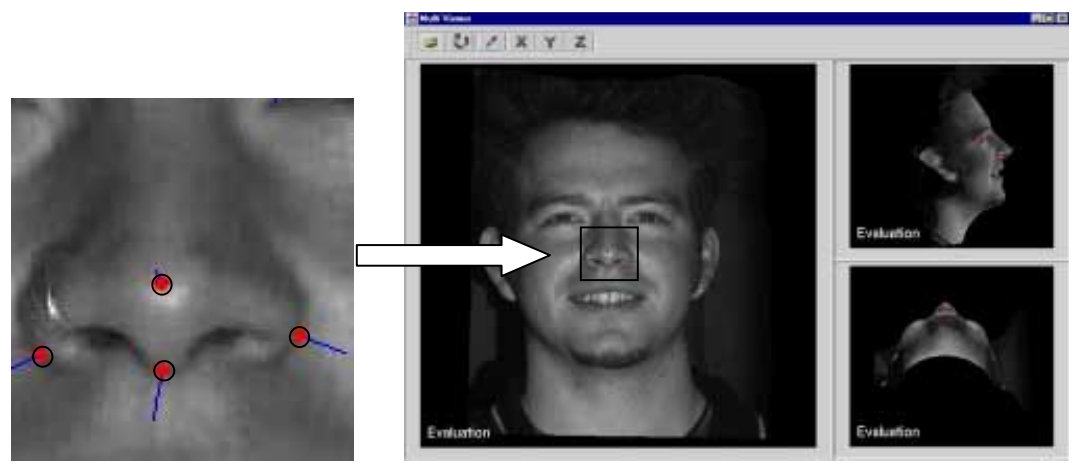

Fig. 2. Graphical interface for facial landmark extraction.

\section{Experimental Results}

The surface registration method - HICP algorithm has been tested using both simulated data and real facial scans. Measurement of surface volume difference was carried out on dummy facial models with known ground truth.

\subsection{Test of Registration Algorithms with Simulated Surface Data}

Comparisons of the performance of HICP algorithm with original ICP algorithm and another modified ICP algorithm developed by Zhang [7] were made using simulated data. Zhang's registration algorithm also considers outlier problem and uses a statistical-based algorithm to computer the weight. We refer Zhang's ICP algorithm as ZICP in the following comparisons.

The simulated data used in the experiment is mathematically constructed using the function $z=x e^{-x^{2}-y^{2}}, x \in[-1,1], y \in[-2,2]$ with intervals of 0.1 in $x$-direction and 0.2 in $y$-direction, generating $4413 \mathrm{D}$ surface points. The surface data is then scaled up by a factor of 25 . This surface was chosen because it has significant variability in gradient over the interval given. Fig 3 plots the simulated surface data. A random rotation along three axes $\omega=3.05, \phi=8.2$ and $\kappa=2.1$ and a translation of $t_{x}=0.51, t_{y}=0.13, t_{z}=-0.26$ are applied to the original surface data to produce the transformed surface (Fig 4).

The performances of the three algorithms under different conditions were compared. All the results and figures given below are repeated and averaged by 20 tries. The registration errors in rotation are measured in degree and errors in translation are given in absolute values with a comparison of data range $x \in[-25,25], y \in[-50,50], z \in[-10.7,10,7]$.

Comparison of Performances under Gaussian Noise. Zero-mean Gaussian noise with a standard deviation equal to 1 is added to the $x-, y-$ and 


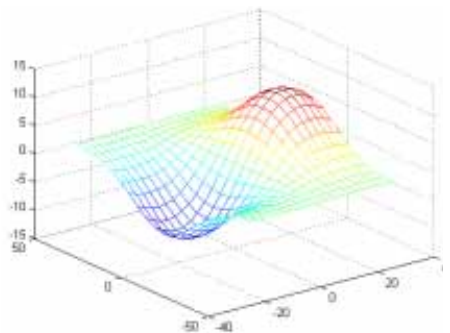

Fig. 3. The simulated original 3D sur- Fig. 4. The transformed 3D surface.

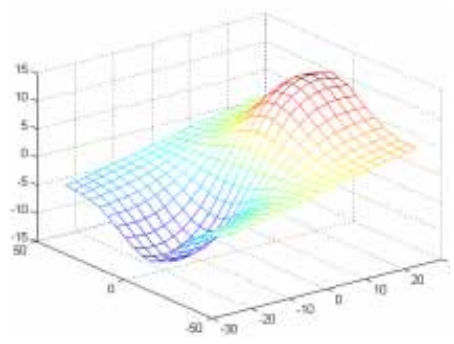
face.

$z$-coordinates of the transformed surface. The reason for chosen Gaussian noise is that the uncertainty in a $3 \mathrm{D}$ point reconstructed from stereo is very similar to Gaussian noise and usually modeled as Gaussian [7]. This noise is then multiplied by a scale factor $c$ to simulate different levels of noise. $C$ ranges from 0 to 1.2 with an incremental interval of 0.2 . Fig 5 plots the registration results from the three algorithms. As we can see, they have similar performances under Gaussian noise.
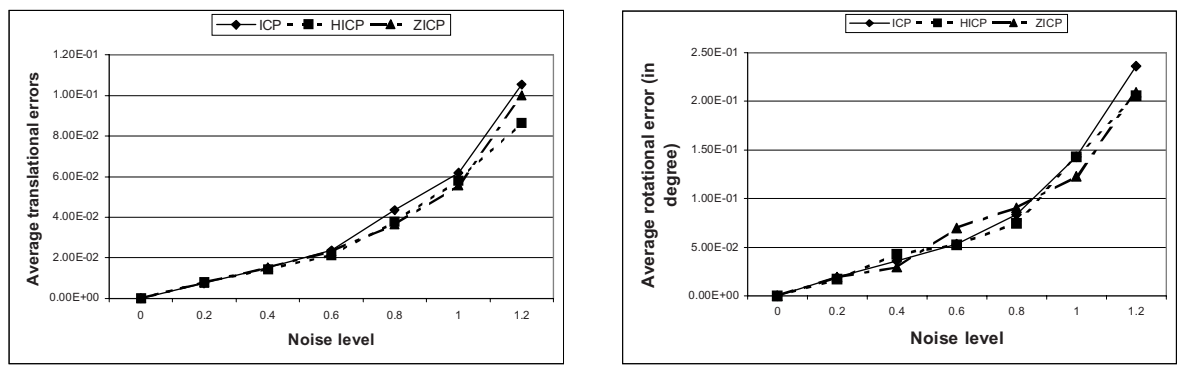

Fig. 5. Performances of three registration algorithms under Guassian noise. Translational and rotational errors as function of noise level.

Comparison of Performances with Outliers. In some cases only a small part of surface data is exposed to noise or has high levels of noise (we usually call it outlier) while other part of data is relatively good. To simulate such situation, uniform noise drawn from $[-2,2]$ is added to part of the transformed data. Fig 6 shows the performances of the three registration algorithms when the percentage of outlier increases. Fig 7 plots the rates of convergence (number of iterations required) when the changes of root mean square (RMS) distance between the original surface and the transformed surface falls below a preset threshold (1e-10). In this case, our algorithm (HICP) has the best performance and the fastest convergence rate, especially when the percentage of ourlier is less than $50 \%$ which is usually the case in real 3D facial scans. 

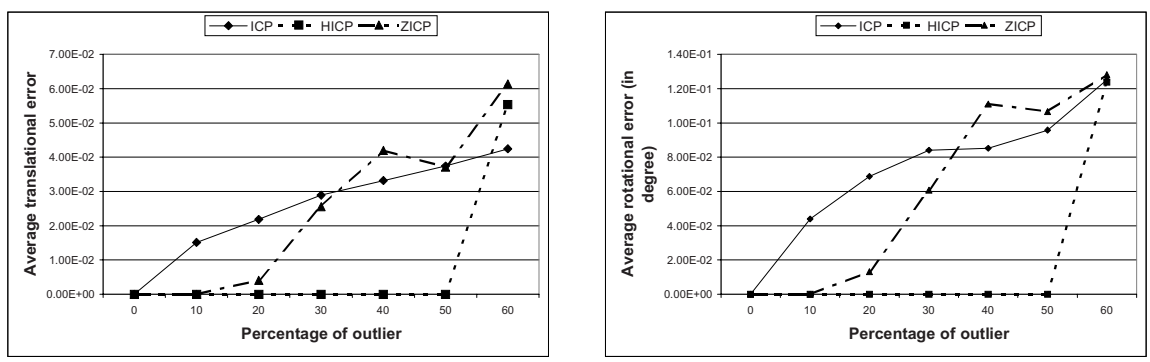

Fig. 6. Performances of the registration algorithms as a function of percentage of outlier.

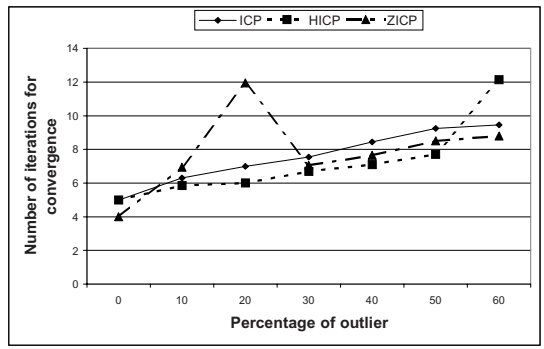

Fig. 7. The rates of the registration algorithms as a function of percentage of outlier.

Comparison of Performances Related to the Region of Overlap. The worst case in registering real surface is that the two surface patches are only partly overlapping and noise is also present. In the third test, zero-mean Gaussian noise with a standard deviation of 0.5 is added to the $x-, y-$ and $z$-coordinates of the transformed surface to simulate the presence of noise. At the same time, we vary the percentage of overlap between two surface patches. Fig 8 shows the performances of the three algorithms under this situation and Fig 9 plots the rate of convergence as the percentage of overlap decreases. The HICP algorithm has the best performance.

As has been shown from the above three tests using the simulated surface data, our surface registration algorithm - HICP has the overall best performance. It's especially robust in the presence of outlier and when the two surfaces to be aligned are only partially overlapped. The convergent speed of HICP is also stable and better than the other two algorithms.

\subsection{Test of HICP Using Real Facial Scans}

HICP algorithm is also validated using real facial scans captured at different times. Two case studies are reported. 

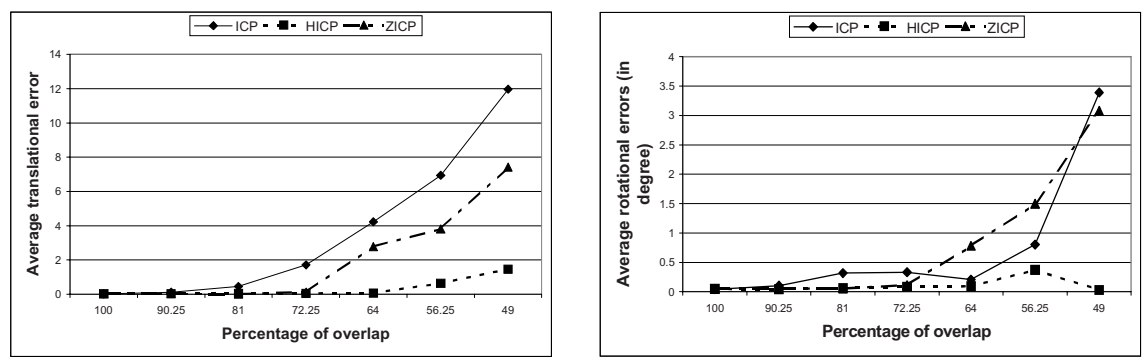

Fig. 8. Performances of the registration algorithms as a function of percentage of overlap.

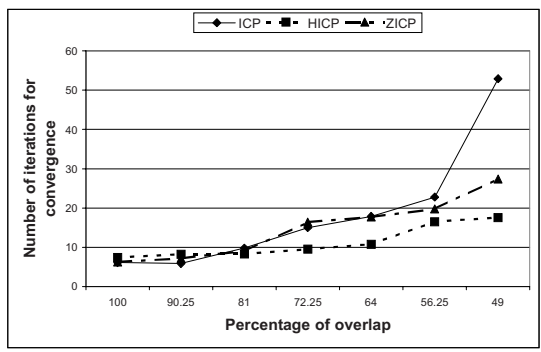

Fig. 9. The rates of the registration algorithms as a function of percentage of overlap.

Case 1: registration of the $3 D$ facial models of the same person captured at different times.

The face of a same person was scanned using the C3D system at different positions. The 3D facial models were generated at a resolution of $5 \mathrm{~mm}$. Fig 10 (a) displays the two facial models before registration, one with solid surface and the other with triangle mesh. The surface patch selected for registration is displayed as meshes in Fig 10 (b). The poses of these two models were significantly different before registration, and the RMS between closest point pair was $51.056 \mathrm{~mm}$. After 50 iterations using HICP algorithm, RMS was reduced to $1.773 \mathrm{~mm}$ and the aligned surfaces is shown in Fig 11.

Case 2: Measure facial growth using controlled facial models.

To simulating facial growth, a dummy facial model is scanned (Fig 12. (a)) and then material was applied to the face (Fig 12 (b)). It produces similar effect to facial growth. The ground truth for the volume added in is $11.533 \mathrm{~cm}^{3}$. Both models were produced at a resolution of $2 \mathrm{~mm}$ by the C3D system and the HICP algorithm was used to align them. After 28 iterations the algorithm converged at a RMS distance of $1.0 \mathrm{~mm}$. A Volume difference of $11.679 \mathrm{~cm}^{3}$ was obtained based on the aligntment. Comparing with the ground truth, the difference is only $1.3 \%$. 


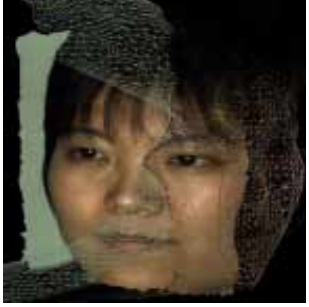

(a) Initial facial positions.

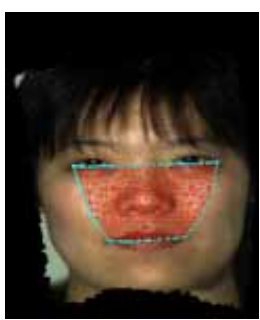

(b) Region for registration.
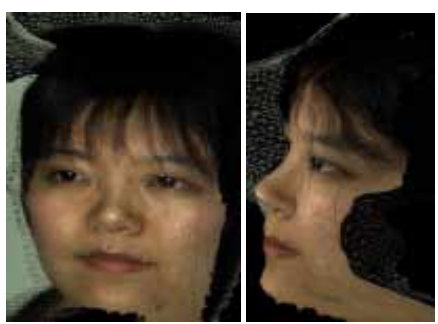

Fig. 11. Facial surfaces after registration.

Fig. 10. Facial surfaces after registration.

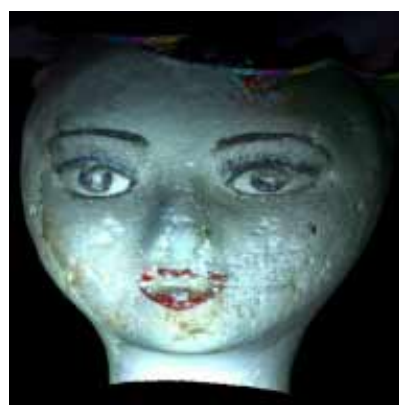

(a) before facial growth

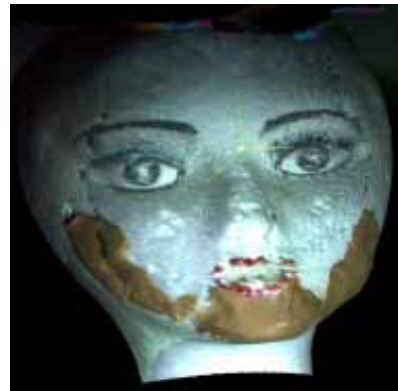

(b) after facial growth

Fig. 12. Dummy facial model for the simulation of facial growth

\section{Discussion and Further Work}

In this paper, techniques for capturing 3D facial models and measuring facial soft tissue changes are presented. A new registration algorithm - HICP algorithm is developed to align 3D facial surface. This algorithm was tested and compared with other registration algorithms using simulated data. The results show that our algorithm has better performance when outlier is present or when the two surface patches are only partially overlapped. Experimental results on the real facial scans and controlled model indicate that the C3D system and the proposed measurement techniques are reliable and capable of detecting small changes in facial soft tissue morphology.

However, this work is still in its initial stage and clinical data collection is still being carried out. When data capture is completed, statistical shape analysis method, such as Procrustes analysis [8], Principal Component Analysis (PCA), Euclidean Distance Matrix Analysis (EDMA) [9] and Thin-plate Spline 
(TPS) visualisation [10] will be used to measure facial growth and to establish statistical models for normal faces and deformed faces due to facial cleft. We believe that by applying 3D capture, analysis and measurement techniques, a more comprehensive understanding of the effect of facial cleft/palate repair on facial soft tissue morphology will be achieved.

\section{References}

1. Besl, P.J. and McKay, N.D. (1992) A method for registration of 3D shapes, IEEE Transactions on Pattern Analysis and Machine Intelligence, 14(2), 239-256.

2. Dryden, I. L. and Mardia, K. V. (1998)Statistical shape analysis, London, John Viley.

3. Ayoub, A. F., Siebert, P., Moos, K. F., Wray, D., Urquhart, C. and Niblett, T. B. (1998) A vision-based three-dimensional capture system for maxillofacial assessment and surgical planning, British Journal of Oral and Maxillofacial Surgery, 36, 353-357.

4. Arun, K.S., Huang, T.S. and Blostein, S.D. (1987) Least-squares fitting of two 3D point sets, IEEE Transactions on Pattern Analysis and Machine Intelligence, 9(5), 698-700.

5. Haralick, R. M., Joo, H., Lee, C. N., Zhuang, X., Vaidya, V. G. and Kim, M. B. (1989) Pose estimation from corresponding point data, IEEE Transactions on Systems, Man and Cybernetics, 19(60), 1426-1446.

6. Mao, Z. and Nafte, A. (1998) 3D image analysis of facial shape changes using depth from stereo, Proceedings of Noblesse Workshop on Non-Linear Model Based Image Analysis, 1-3 July, Glasgow, UK, Springer, pp.283-288.

7. Zhang, Z. (1994) Iterative point matching for registration of free-form curves and surfaces, International Journal of Computer Vision, 13(2), 119-152.

8. Rohlf, F. J. and Slice, D. (1991) Extensions of the Procrustes method for the optimal superimposition of landmarks, Systematic Zoology, 39(1), 40-59.

9. Lele, S. (1993) Euclidean distance matrix analysis (EDMA): estimation of mean form and mean form difference, Mathematical Geology, 25, 573-602.

10. Bookstein, F. L. (1989) Principal warps: thin-plate spline and the decomposition of deformations, IEEE Transactions on Pattern Analysis and Machine Intelligence, 11(6), $567-585$. 